\title{
Growth dynamics of microbubbles on microcavity arrays by solvent exchange: Experiments and numerical simulations
}

\author{
Shuhua Peng ${ }^{\mathrm{a}, \mathrm{b}, *}$, Vamsi Spandan ${ }^{\mathrm{c}}$, Roberto Verzicco ${ }^{\mathrm{c}}$, Detlef Lohse ${ }^{\mathrm{c}, \mathrm{d}}$, Xuehua Zhang ${ }^{\mathrm{e}, \mathrm{a}}$ \\ ${ }^{a}$ Soft Matter E Interfaces Group, School of Engineering, RMIT University, Melbourne, VIC 3001, Australia \\ ${ }^{\mathrm{b}}$ School of Mechanical and Manufacturing Engineering, University of New South Wales, NSW 2031, Australia \\ ' Physics of Fluids Group, Department of Science and Technology, Mesa+ Institute, J.M. Burgers Centre for Fluid Dynamics, University of Twente, P.O. Box 217, 7500 AE Enschede, \\ The Netherlands \\ ${ }^{\mathrm{d}}$ Max Planck Institute for Dynamics and Self-Organization, 37077 Göttingen, Germany \\ e Department of Chemical and Materials Engineering, University of Alberta, Edmonton, Alberta T6G 1H9, Canada
}

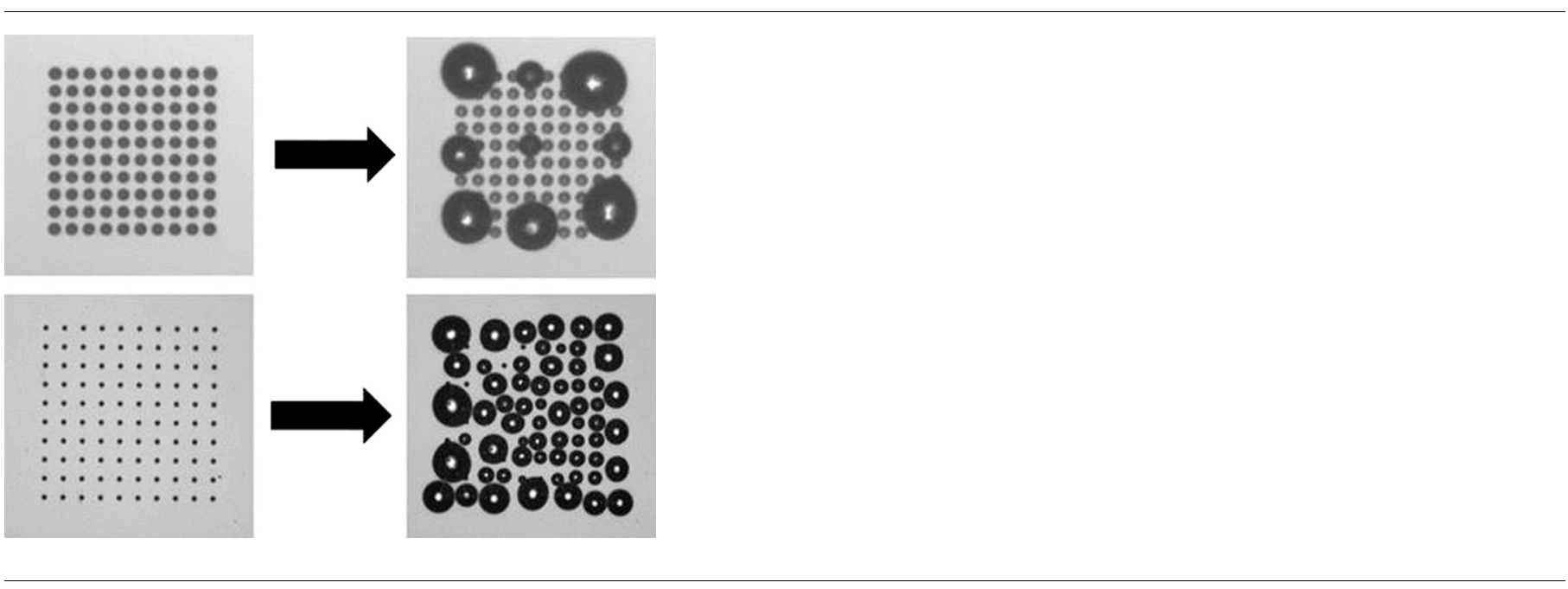

\section{A R T I C L E I N F O}

\section{Article history:}

Received 16 April 2018

Revised 24 July 2018

Accepted 25 July 2018

Available online 27 July 2018

\section{Keywords:}

Solvent exchange

Surface nanobubbles

Growth dynamics

Hydrophobic cavity arrays

\begin{abstract}
A B S T R A C T
Solvent exchange is a flow process to induce a transient oversaturation for forming nanobubbles or nanodroplets on solid surfaces by displacing the solution of gases or droplet liquids with a controlled flow of a poor solvent. In this work, we experimentally and numerically investigate the effect of the flow rate and other control parameters on the formation of microbubbles on hydrophobic cavity arrays during the solvent exchange process. We find that the growth rate, location, and number density of microbubbles are closely related to flow rate, solvent concentration, cavity distance, and spatial arrangement. Higher growth rates and number densities of the bubbles were obtained for faster solvent exchange flow rates. The competition of neighbouring growing bubbles for dissolved gas is greatly alleviated when the intercavity distance is increased from $13 \mu \mathrm{m}$ to $40 \mu \mathrm{m}$. The effects of the flow rate and the cavity spacing on the bubble growth are in agreement with the observations from our three-dimensional numerical simulations. The findings reported in this work provide important insight into the formation of multiple interacting surface microbubbles under various flow conditions. The understanding may be extended to a
\end{abstract}

\footnotetext{
* Corresponding author at: School of Mechanical and Manufacturing Engineering, University of New South Wales, NSW 2031, Australia.

E-mail addresses: shuhua.peng@outlook.com (S. Peng),xuehua.zhang@ualberta.ca (X. Zhang).
} 
smaller scale for the growth of surface nanobubbles during solvent exchange, which is much harder to visualize in experiments.

(c) 2018 Elsevier Inc. All rights reserved.

\section{Introduction}

Nucleation and growth of bubbles on a solid surface is ubiquitous in many physical and chemical processes. Often the bubbles grow big enough and detach from the surface. However, there are several processes where surface nanobubbles (i.e. nanoscopic gaseous domains on immersed substrates) can be produced and observed by techniques with high spatial resolution [1-11]. The simplest way to generate surface nanobubbles is by directly immersing a hydrophobic substrate into air-equilibriated water or by depositing a water droplet onto a hydrophobic surface [12]. Spontaneous formation of surface nanobubbles takes place during the immersion process. Production of surface nanobubbles by photochemical and electrochemical reactions has also been carried out [13-15,11,16-19]. In a typical electrochemical reaction, a large amount of gas molecules is produced in a short amount of time and hence high gas concentration is built up in the system, which leads to bubble nucleation and formation on the surfaces of reactors and electrodes. Interestingly, White et al. investigated the formation of bubbles on an electrolyte as small as tens of nanometers where the current reflects the nucleation events under potential [20].

One of the earliest approaches to produce surface nanobubbles is called solvent exchange, where a good solvent of gas is slowly replaced by a poor one and the resulting gas oversaturation leads to formation of nanobubbles on the immersed substrates [21,22,17,23-26]. As adapted by several different research groups, solvent exchange has been successfully employed to produce surface nanobubbles on various substrates such as hydrophobic silicon surface, HOPG, and mica [27-31]. Moreover, solvent exchange is also applied to produce nanodroplets of many kinds of liquids on solid surfaces. Progress has been made recently to quantitatively understand nanodroplet formation by solvent exchange. Essentially a pulse of oversaturation is created when the solution of the droplet liquid is displaced by a poor solvent, which drives the nucleation and growth of nanodroplets. The growth rate, final size and spatial distribution of droplets are governed the flow rate, the channel dimensions and the solution composition in the ternary solubility phase diagram [32].

However, rather limited research has been conducted to understand the growth dynamics of interacting bubbles under different flow conditions. Recent work showed that for a given flow rate, the growth of bubbles on microcavities is profoundly influenced by the inter-cavity spacing, because of competition effects between neighbouring bubbles [33]. But it is not yet clear how the growth dynamics and the collective interaction of the bubbles are coupled with the flow rate of the solvent exchange process.

For the formation of surface nanodroplets, recent work has shown that the flow rate of the solvent exchange is one of the most important parameters that influences the growth dynamics of the droplets [34]. Both theoretical analysis and experimental measurements by total internal reflection microscope confirm that the growth time scales with the square root of the Peclect number (the dimensionless flow rate) of the displacing solvent [35]. The flow rate therefore has significant influence on the size and number density of the droplets. Again, the effect of flow rate on the microbubble formation has much less been analyzed, although the understanding will be necessary for controlled formation of microbubbles by solvent exchange.

In this work, we will fill the gap and study the effect of flow conditions on the formation of microbubbles by solvent exchange. Cavity substrates with microcavity arrays are designed to determine the location for bubble formation. The flow rate, solvent concentration, patterned cavity distance and arrangement will be varied in the experiments. In addition, we perform threedimensional numerical simulations to study the effects of the solvent exchange flow rate and cavity arrangement on the initial stages of the solvent-exchange induced bubble growth, finding reasonable agreement with the experiments. The numerical scheme is based on the immersed boundary method (IBM) [36,37]. The objective of the simulations is threefold: (1) These simulations can be used to reveal the physical mechanisms of the process. (2) They can be applied to situations that are less easily or not all accessible in experiments. They then provide a predictive tool. (3) They show that even the complex process of solvent exchange can be numerically modelled, as seen by the reasonable agreement with experiment data. The findings in this work thus provide insight into the controlled formation and growth of microbubbles by solvent exchange. The results can potentially be applied to the formation and diffusive interaction of smaller nanobubbles.

\section{Experimental and numerical section}

\subsection{Experimental setup}

1H,1H,2H,2H-perfluorodecyltriethoxysilane (PFDTS) was purchased from Sigma-Aldrich. Chemicals including sulfuric acid (98\%), hydrogen peroxide (35\%), and ethanol (100\%) were from Merck Pty Ltd. All chemicals were used without further purification. Details for the fabrication of hydrophobic microcavity arrays and procedures for bubble growth by solvent exchange have been given in our previous work [33]. Briefly, microcavity arrays on silicon substrates were fabricated by deep reactive-ion etching (DRIE) and subsequently hydrophobilized by coating a layer of PFDTS by chemical vapor deposition (CVD). The spacing between the cavities and the arrangement of the cavities were determined by the design of the masks.

For the microbubble formation by solvent exchange, the microchannel with hydrophobic microcavity arrays was first filled with air-saturated water, which was then slowly replaced by ethanol solution using a syringe pump. Already in the filling process, air was trapped in the cavities upon contact with water. During solvent exchange, the trapped air bubbles in the cavities grew in response to the oversaturation, as ethanol was introduced. It should be noted that the order for the injection of water and ethanol was opposite for the conventional process for surface nanobubble formation by solvent exchange. The bubble growth was in situ monitored by an upright optical microscope with long working distance objective with 10 times magnification and the experiment setup is illustrated in Fig. 1. The top-view of the bubbles was recorded at the speed of 10 frames per second. The maximal bubble radius was analyzed by image processing in Matlab. The flow conditions in the experiments are listed in Table 1. 


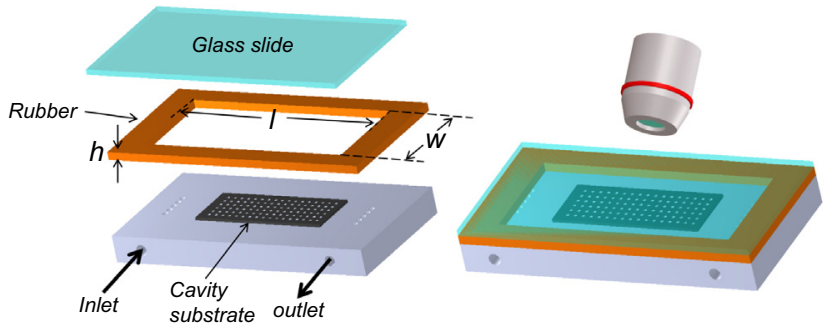

Fig. 1. Scheme of experimental setup for the in situ observation of bubble growth during solvent exchange.

Table 1

Experimental conditions for bubble formation and growth by solvent exchange on cavity surface. $Q$ is the flow rate, $R e$ is the Reynolds number $(R e=Q /(w v), v$ is viscosity), $P e$ is the Peclet number $(P e=Q /(w D), D$ is diffusivity), $h, l$ and $w$ are the height, length, and width of the fluid channels, respectively.

\begin{tabular}{lccccc}
\hline$Q(\mu \mathrm{L} / \mathrm{min})$ & $R e$ & $P e$ & $h(\mathrm{~mm})$ & $l(\mathrm{~mm})$ & $w(\mathrm{~mm})$ \\
\hline 100 & 0.033 & 98 & 2.21 & 60 & 17 \\
200 & 0.066 & 196 & 2.21 & 60 & 17 \\
500 & 0.17 & 490 & 2.21 & 60 & 17 \\
1000 & 0.33 & 980 & 2.21 & 60 & 17 \\
\hline
\end{tabular}

\subsection{Numerical details}

We use direct numerical simulations (DNS) to solve the NavierStokes equations in a three-dimensional Cartesian domain, using a second-order accurate finite-difference scheme with fractional time-stepping. The flow solver is coupled with a versatile moving least squares immersed boundary method (IBM) which enforces the interface boundary conditions on the bubble surface. In IBM, the influence of any immersed body (moving or stationary, rigid or deformable) on the surrounding carrier fluid is imposed through a volume averaged body force included in the governing equations for the carrier fluid. This allows the use of a single mesh for the numerical solution of the carrier fluid throughout the simulation, which immensely reduces the computational cost in comparison to other body-fitted techniques [38].

In order to account for the growth of bubbles through solvent exchange, we solve an advection-diffusion equation for the oversaturation in the flow domain. The computational setup is similar to the one used in our previous work [36]. The boundary conditions for flow domain are as follows: (i) inflow-outflow in the streamwise direction, (ii) no-slip walls in the wall-normal direction, and (iii) periodic in the span-wise direction. Hemispherical bubbles are initialized at the bottom surface in a matrix-like arrangement using spacing ratios similar to that of the experiments. The surface of each bubble is meshed using a triangulated network and the resolution is maintained in such a way that the mean edge length of the triangles is about $0.7-0.9$ times the local carrier fluid grid spacing (required in IBM, see $[39,40]$ ). Through the IBM, a free slip boundary condition is imposed at the interface of the immersed bubble and incoming flow. The over-saturation in the incoming flow is initialized in the form of a Gaussian pulse that is advected in time.

At any given instant, the concentration gradient on the surface of the immersed bubble is computed using the following procedure. First, a numerical probe is sent along the normal of each triangle a distance equal to the mean grid spacing. At the end-point of the probe, we interpolate the concentration from the carrier fluid numerical solution using which we compute the mass flux of the gas into the bubble from each triangular element. For the full bubble, we sum up the mass flux over all triangular elements and this gives us the overall increase in volume of the bubble per time step. Since we constrain the bubble to have a fixed contact area, the positions of individual triangular elements are updated according to the total mass influx. The effect of coalescence is not taken into consideration in the simulations. Instead, the simulation is stopped when the interfaces of neighboring bubbles comes closer than five grid cells, which is the resolution required to approximately resolve the fluid in between the approaching interfaces. The simulations performed in this work give insight on the dynamics of bubble growth during solvent exchange in the initial growing phase of the micro-pit array.

A total of $7 \times 7=49$ bubbles are initialized and allowed to grow under four different flow rates which match the experiments. The grid resolution is $480 \times 240 \times 240$ grid points in the stream-wise, span-wise and wall-normal directions, respectively. The bubbles are initialized using 2592 triangles while adaptive refinement of the triangles is employed when necessary to satisfy the resolution constrain discussed previously. Additional details on the numerical techniques and validation of the code can be found in literatures $[41,39]$.

\section{Results and discussion}

\subsection{Effect of solvent exchange rate}

Four different flow rates were performed to form bubbles on the microcavity arrays by solvent exchange. As shown in Fig. 2(a), bubbles first symmetrically grew at the four corners of the cavity pattern when a slow solvent exchange rate of $100 \mu \mathrm{L} / \mathrm{min}$ was applied. As the solvent exchange processed further, bubbles were then observed on the edges of the cavity array. The term "edge bubbles" used below refers to the bubbles located on the edges of the cavity array with $10 \times 10$ cavities. Then, the slowly formed bubbles on the pattern edges shrunk while the ones at the four corners kept growing. It is rare to observe bubble formation at the middle of the cavity pattern when the solvent exchange rate was 100 $\mu \mathrm{L} / \mathrm{min}$. The bubble growth behaviour was similar when the solvent exchange rate was increased to $200 \mu \mathrm{L} / \mathrm{min}$ (Fig. 2(b)). Bubbles symmetrically grew from the corners and then the edges. We can clearly see that bubbles grew in the inner area of the square cavity pattern when the solvent exchange rate was $200 \mu \mathrm{L} / \mathrm{min}$. This was attributed to the increased oversaturation level at higher solvent exchange rate and the inner cavities grew at this condition. The sequence of the collective bubble growth behaviour has already been discussed in our previous work [33].

The bubble growth behaviour was completely different when the solvent exchange rate was further increased to $500 \mu \mathrm{L} / \mathrm{min}$ (Fig. 2(c)) and $1000 \mu \mathrm{L} / \mathrm{min}$ (Fig. 2(d)), respectively. As shown in Fig. 2(c), the bubbles quickly grew at the front edge of the cavity array along the solvent exchange direction within $3 \mathrm{~s}$ while there was no obvious bubble formation at the rear side of cavity array. As the solvent exchange duration increased to $7 \mathrm{~s}$, the bubbles at the front edge kept growing and then merged into bigger ones and at the same time, the bubbles located at the other edges and the inner area started growing. No significant difference in the bubble size on these two areas was observed at this stage. After that, edge effect dominated the bubble growth and the bubbles at the edges became bigger than the ones in the inner area. The same behaviour of bubble growth was observed when the solvent exchange rate was increased to $1000 \mu \mathrm{L} / \mathrm{min}$ (Fig. 2(d)). We can clearly see that the symmetric arrangement of bubbles was not preserved at the high solvent exchange rates while the edge effect still played a predominated role for the bubble growth at the late stages.

The corresponding mean radius of the growing bubbles and the bubble number as a function of solvent exchange time (the time 
(a)

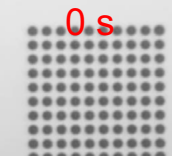

(b)

(c)

(d)

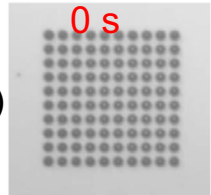

S

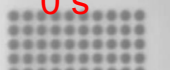

t:t:


3
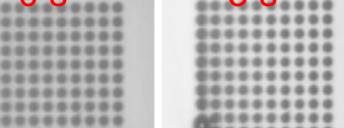

(1)
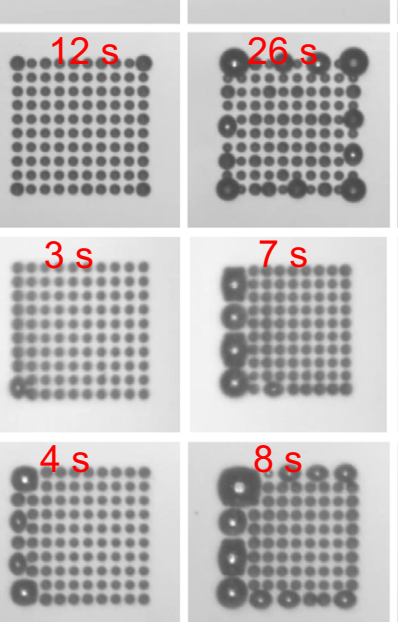
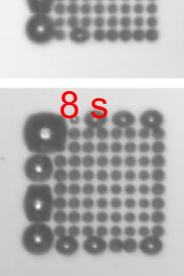
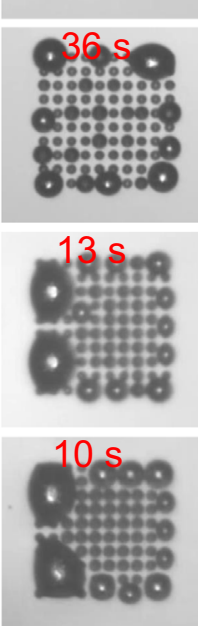
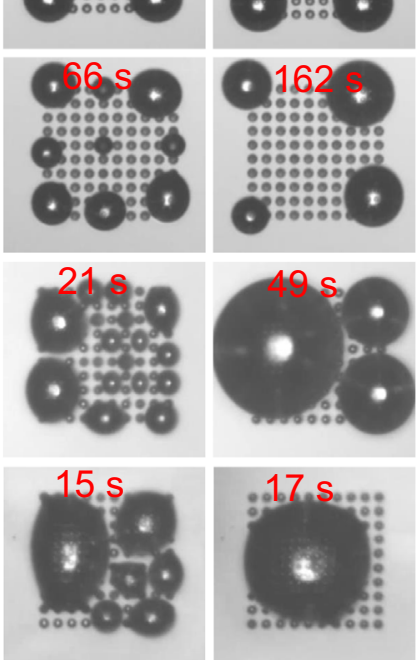

$100 \mu \mathrm{m}$


(b) $200 \mu \mathrm{L} / \mathrm{min}$; (c) $500 \mu \mathrm{L} / \mathrm{min}$; and (d) $1000 \mu \mathrm{L} / \mathrm{min}$. The solvent exchange time is indicated.

after the beginning of the solvent exchange) is shown in Fig. 3. The growth rate of the bubble radius is between $0.1 \mu \mathrm{m} / \mathrm{s}$ up to $0.7 \mu \mathrm{m} / \mathrm{s}$, respectively when the solvent exchange was conducted at the rate of $100 \mu \mathrm{L} / \mathrm{min}$ to $1000 \mu \mathrm{L} / \mathrm{min}$. Clearly, fast solvent exchange rate results in fast growing bubbles. This trend is essentially the same for nanodroplet formation by solvent exchange [42].

We note that the exact function of the bubble size with time in general and for longer times is much more complicated than a
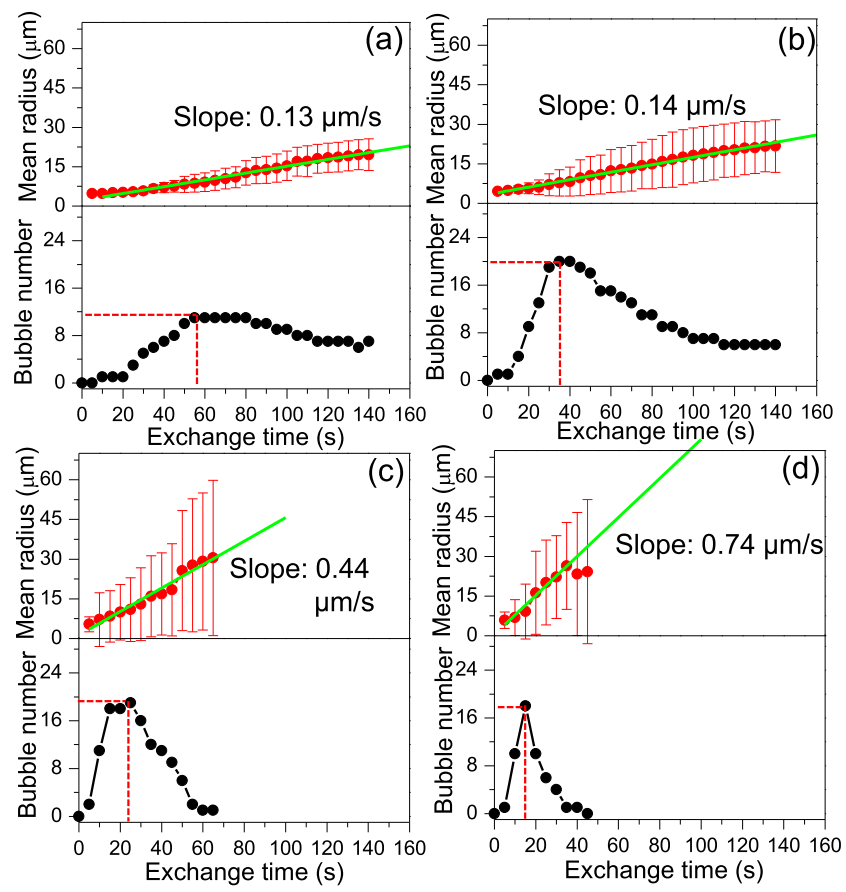

Fig. 3. Corresponding mean radius and number of growing bubbles under different flow rates: (a) $100 \mu \mathrm{L} / \mathrm{min}$; (b) $200 \mu \mathrm{L} / \mathrm{min}$; (c) $500 \mu \mathrm{L} / \mathrm{min}$; and (d) $1000 \mu \mathrm{L} / \mathrm{min}$. linear increase. If we assume that the effect of the Laplace pressure on the microbubble growth can be neglected, the temporal dependence of the contact angle $(d(\theta) / d t)$ of a bubble in a constant contact area growth mode approximately follows the same relationship as derivated for surface nanodroplets, see our previous work on the nanodroplet formation by solvent exchange [43], namely

$\frac{d \theta}{d t} \sim \frac{4 D P e}{\rho_{d} L^{2}}(1+\cos \theta)^{2} f(\theta) c_{s}(t) \zeta(t)$

where $D$ is the diffusion coefficient, $P e$ is the Peclet number, $\rho_{d}$ is density, $L$ is the cavity diameter, $c_{s}(t)$ is the time dependent saturation concentration during the solvent exchange process, $c_{s}(t) \zeta(t)$ is the oversaturation pulse. A geometry factor, $f(\theta)$, is a function of the contact angle of the bubble

$f(\theta)=\frac{\sin \theta}{1+\cos \theta}+4 \int_{0}^{\infty} \frac{1+\cosh 2 \theta \xi}{\sinh 2 \pi \xi} \tanh [(\pi-\theta) \xi] d \xi$

From the bubble contact angle $(\theta)$ and the diameter of the microcavities $(L)$, the bubble radius $(R)$ can be obtained by a simple geometric analysis $\left(R=\frac{L}{2 \sin \theta}\right)$ [33]. However, in a small time window, the growth rate can always be approximated to be linear.

The number of growing bubbles (in short, 'bubble number') increased at the first stage of solvent exchange and then decreased due to depletion effects and bubble coalescence. The faster the solvent exchange rate, the shorter the time required to achieve the maximum bubble number. The maximum bubble number increased from 10 to 20 when the solvent exchange rate was changed from 100 to $200 \mu \mathrm{L} / \mathrm{min}$. More microcavities were activated for bubbles to grow when a higher oversaturation level was achieved at $200 \mu \mathrm{L} / \mathrm{min}$ flow rate condition. The maximum bubble number decreased to 19 and 18 , respectively, as the solvent exchange rate increased to $500 \mu \mathrm{L} / \mathrm{min}$ and $1000 \mu \mathrm{L} / \mathrm{min}$. Coalescence of the fast growing bubbles at the front edge of the pattern array was observed at higher flow rate conditions. 
The bubble formation and growth is also affected by the gas solubility in the solutions. The solvent exchange was carried out by replacing water with $70 \%$ ethanol solution rather than pure ethanol. As shown in Fig. 4, under the same corresponding solvent exchange rate, the slopes for the mean bubble radius as a function of solvent exchange time decreased accordingly. As discussed in our previous work,[33] after the pure ethanol was replaced by $70 \%$ ethanol, the oversaturation level during solvent exchange
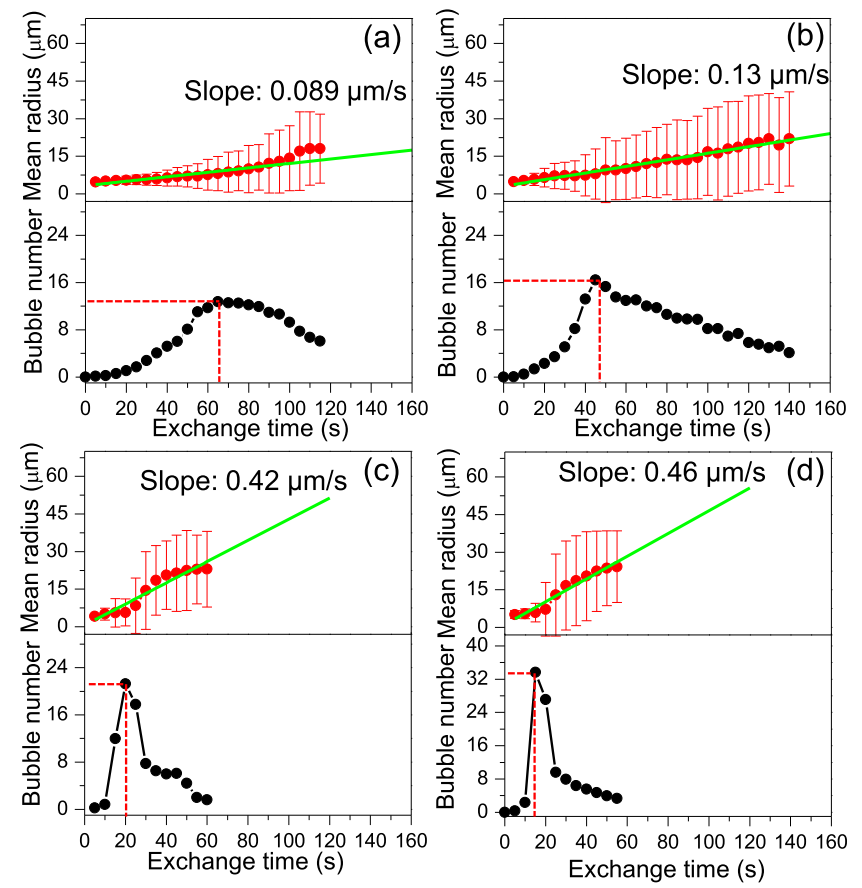

Fig. 4. Bubble formation and growth by exchanging water with $70 \%$ ethanol aqueous solution under different flow rates: (a) $100 \mu \mathrm{L} / \mathrm{min}$; (b) $200 \mu \mathrm{L} / \mathrm{min}$; (c) $500 \mu \mathrm{L} / \mathrm{min}$; and (d) $1000 \mu \mathrm{L} / \mathrm{min}$. decreased since the air solubility in $70 \%$ ethanol is lower than that in pure ethanol. Hence, the bubble growth, which is governed by the air oversaturation level, was retarded. At the same time, the maximum bubble number during solvent exchange did not show notable change for the first three solvent exchange rates $(100 \mu \mathrm{L} / \mathrm{min}, 200 \mu \mathrm{L} / \mathrm{min}$, and $500 \mu \mathrm{L} / \mathrm{min}$, respectively) although slightly longer exchange time was required to achieve the maximum bubble numbers. As for the solvent exchange rate of
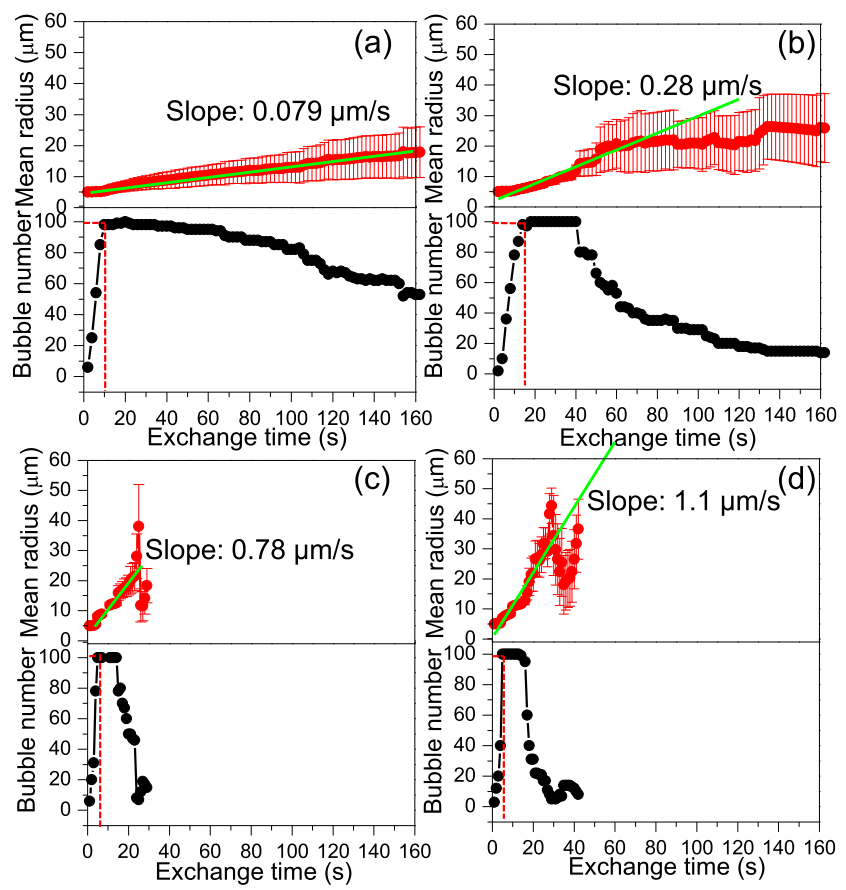

Fig. 6. Corresponding mean radius and number of growing bubbles on loosely packed pattern array under different flow rates: (a) $100 \mu \mathrm{L} / \mathrm{min}$; (b) $200 \mu \mathrm{L} / \mathrm{min}$; (c) $500 \mu \mathrm{L} / \mathrm{min}$; and (d) $1000 \mu \mathrm{L} / \mathrm{min}$.

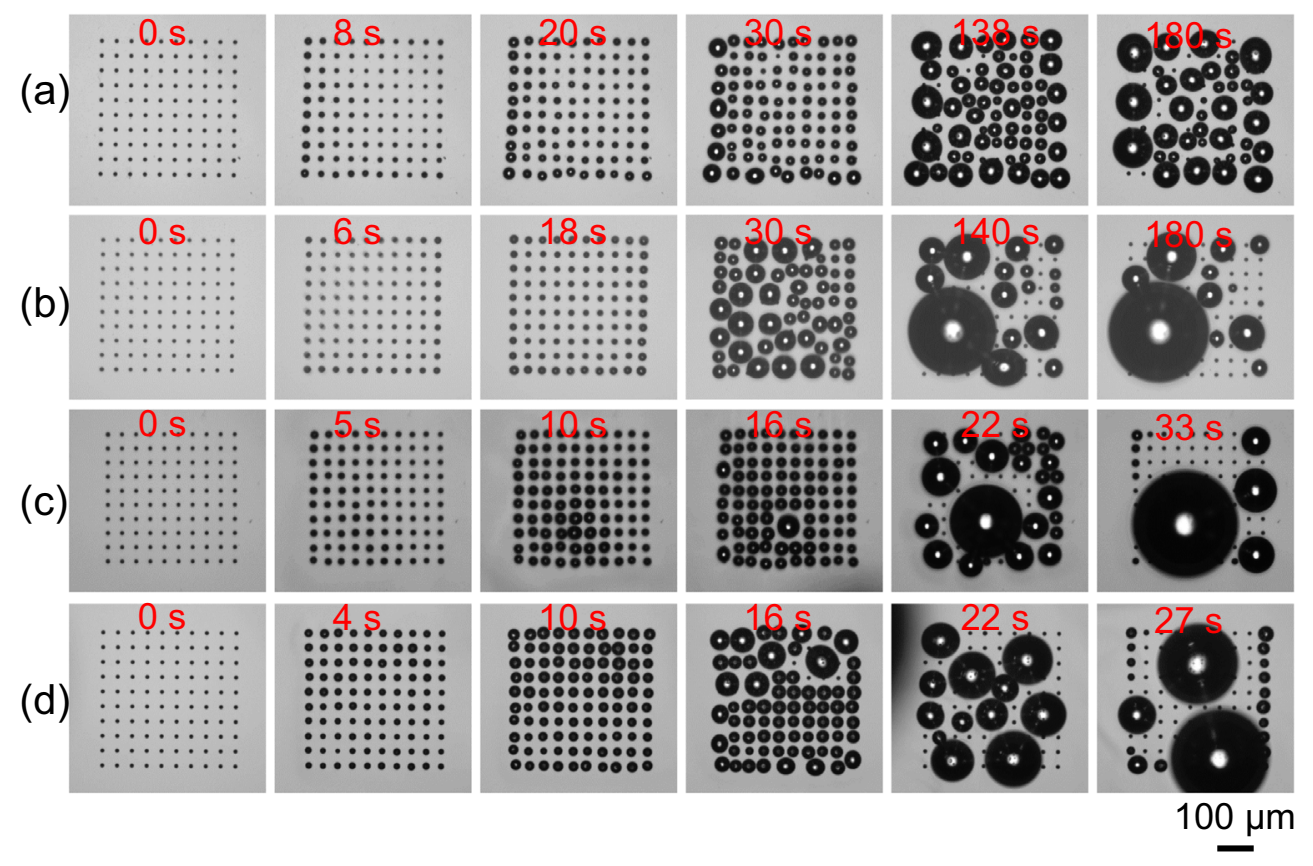

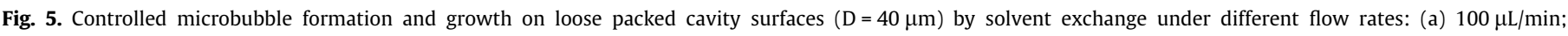
(b) $200 \mu \mathrm{L} / \mathrm{min}$; (c) $500 \mu \mathrm{L} / \mathrm{min}$; and (d) $1000 \mu \mathrm{L} / \mathrm{min}$. The solvent exchange time is indicated. 
$1000 \mu \mathrm{L} /$ min with $70 \%$ ethanol, the combination of moderate bubble coalescence and appropriate oversaturation level during the solvent exchange resulted in the maximum bubble number to increase from 18 to 33 .

\subsection{Effect of pattern array size}

Control of bubble growth can also be carried out by using spatial arrangement of the microcavity arrays. When the cavity distance (centre to centre) increased from $13 \mu \mathrm{m}$ to $40 \mu \mathrm{m}$, the growth rate, number, and location of bubbles exhibited a completely different behaviour. The corresponding in situ snapshots of the bubbles on the loosely packed arrays at different solvent exchange rates are shown in Fig. 5. No symmetric arrangement of the growing bubbles on the pattern array was observed at all four solvent exchange rates. As shown in Fig. 5(a), the edge effect with the larger bubbles at the edges was clearly seen between $\mathrm{t}=20$ to $30 \mathrm{~s}$ and it gradually faded away at later stages and large bubbles in the inner area of the pattern were also observed. The edge effect was further reduced when the solvent exchange rate was increased to $200 \mu \mathrm{L} / \mathrm{min}$ (Fig. 5(b)) and $500 \mu \mathrm{L} / \mathrm{min}$ (Fig. 5(c)), since the largest bubbles on the pattern array were observed in the inner area rather than at the edges. Nevertheless, bubbles grew faster when higher flow rates were applied for solvent exchange, which is consistent with the results for the closely packed pattern.

The detailed analysis of the bubble mean radius and the bubble number as a function of solvent exchange time is given in Fig. 6. The bubble growth rate was between $0.08 \mu \mathrm{L} / \mathrm{s}$ to $1 \mu \mathrm{L} / \mathrm{s}$ when the solvent exchange rates increased from $100 \mu \mathrm{L} / \mathrm{min}$ to $1000 \mu \mathrm{L} / \mathrm{min}$. As expected, higher solvent exchange rate resulted in faster bubble growth rate. An interesting finding here is that all the microcavities on the loosely packed pattern were active (i.e., showed growing
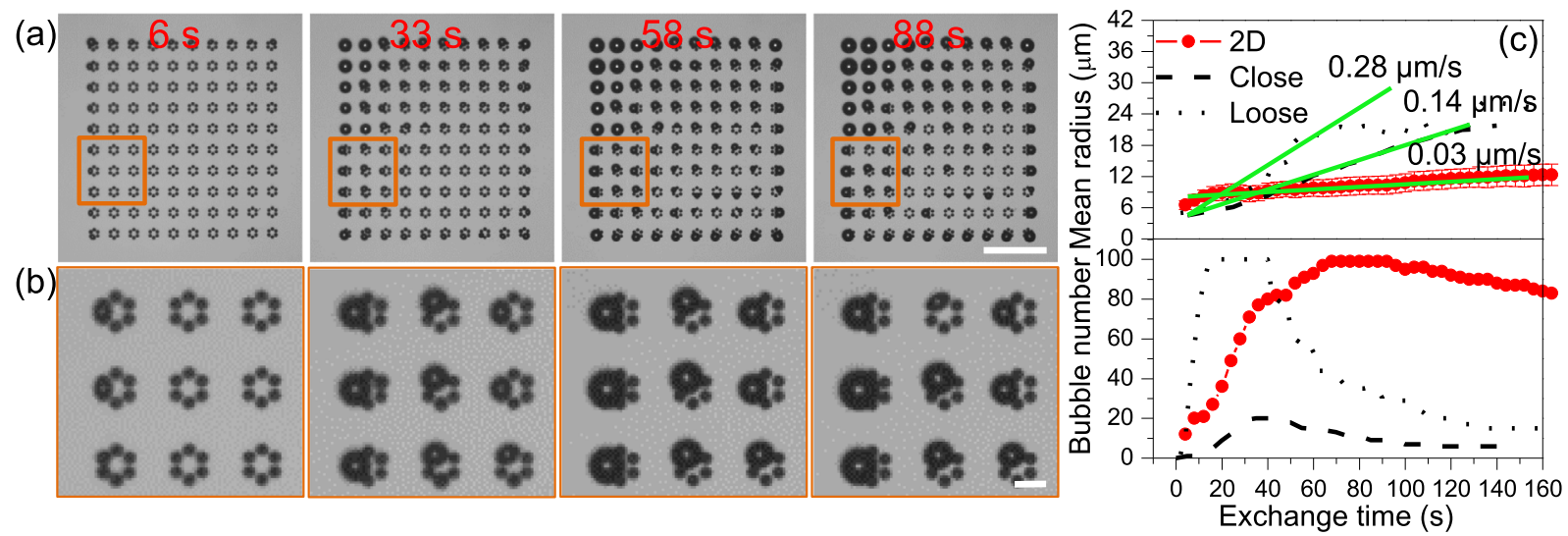

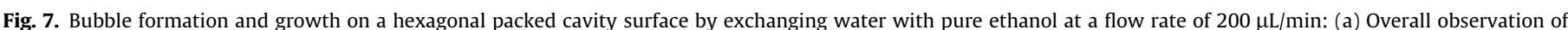

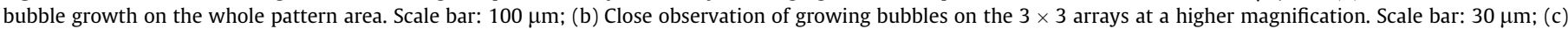
Comparison of bubble growth in terms of mean bubble radius and bubble number on different cavity surfaces under the same flow rate of $200 \mu \mathrm{L} / \mathrm{min}$.

(a)
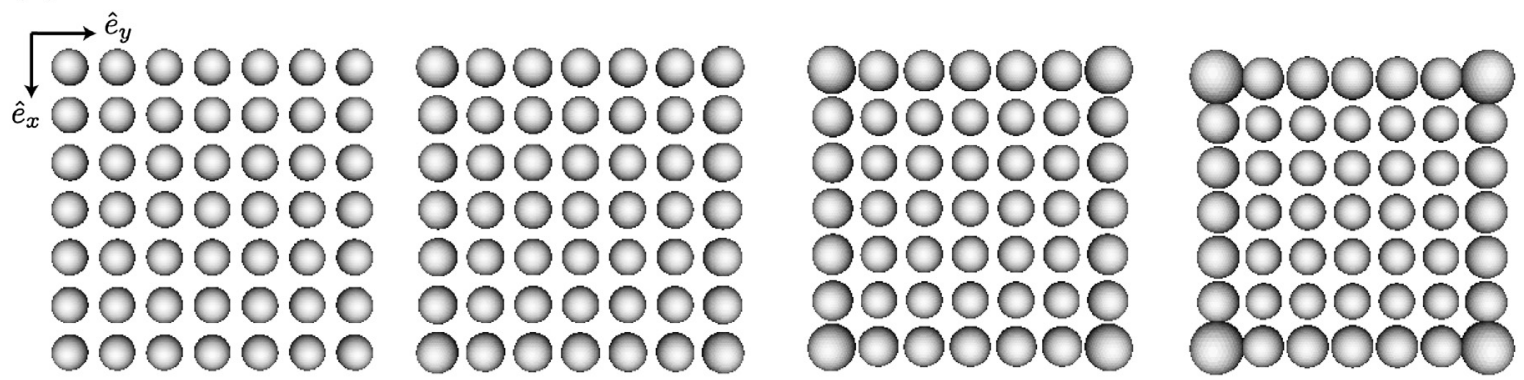

(b)
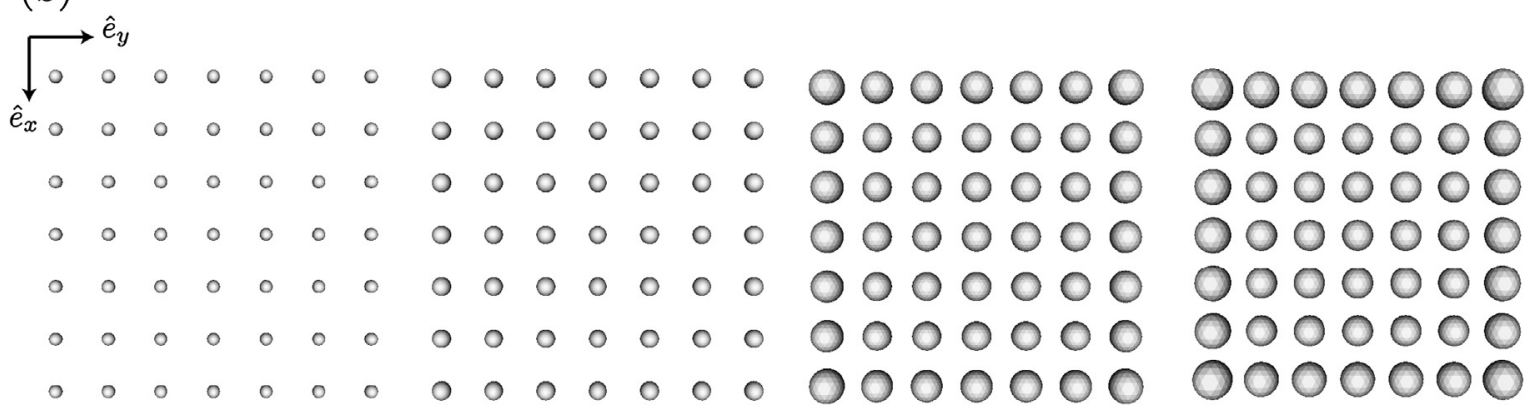

Fig. 8. Instantaneous snapshots of bubbles growing in (a) closely packed array, (b) loosely packed array. 
bubbles) during solvent exchange. Under the above four different exchange rates, the maximum bubble number reached the full 100 , i.e., is identical to the total number of microcavities on the pattern array. The reason is that, as the cavity spacing increased, individual growing bubbles were given enough space to expand before coalescence. At the same time, the depletion effect was reduced as the distance between big bubbles and small ones increased. It can be clearly seen that the distance between cavities has significant influence on the growth behaviour.

\subsection{Bubble growth on hexagonal cavity array}

We now show bubble formation and growth on a hexagonal cavity cluster pattern array, which combines the spatial features of closely and loosely packed cavities previously studied. The pattern compromised cavities closely packed in a hexagonal arrangement as clusters, which were then loosely organized in a square pattern. As shown in Fig. 7(a), under $200 \mu \mathrm{L} / \mathrm{min}$ flow rate condition, the edge effect was clearly observed with the bubbles at the edges growing faster than the ones in the inner area. At the same time, all the clusters on the whole pattern area were covered with growing bubbles due to their extended spacings. A close look of growing bubbles on the hexagonal clusters in Fig. 7(b) revealed that only one out of six cavities was activated for bubble growth, which is similar for the bubbles on the closely packed cavity pat- tern. Fig. 7(c) summarizes the bubble growth behaviour in terms of growing bubble radius and bubble number on three different cavity surfaces under the same flow rate of $200 \mu \mathrm{L} / \mathrm{min}$. Together with the above results on closely and loosely packed cavity surfaces, we can conclude that location, size, growth rate, and number density of bubbles are all controlled by manipulation of solvent exchange conditions and cavity array spacings.

Noticeably, in our experiments we were not able to obtain uniform size of bubbles on the array by solvent exchange even when the inter-cavity distance is already 4 times larger than the cavity diameter. This observation is opposite to nanodroplet formation on chemically patterned surfaces where highly uniform droplet sizes can be obtained by solvent exchange. One possible reason is that the bubble growth may be more sensitive to any fluctuation in the system. Small variation in the local composition and solution composition may be sufficient to lead to large differences in bubble size, due to their much lower density and faster volume expansion, compared to liquid droplets.

\subsection{Numerical study}

We conduct simulations to investigate the effect of cavity distance on the bubble growth behaviour on closely and loosely packed arrays, respectively. In Fig. 8, we show instantaneous snapshots of bubbles growing in both closely packed and loosely packed
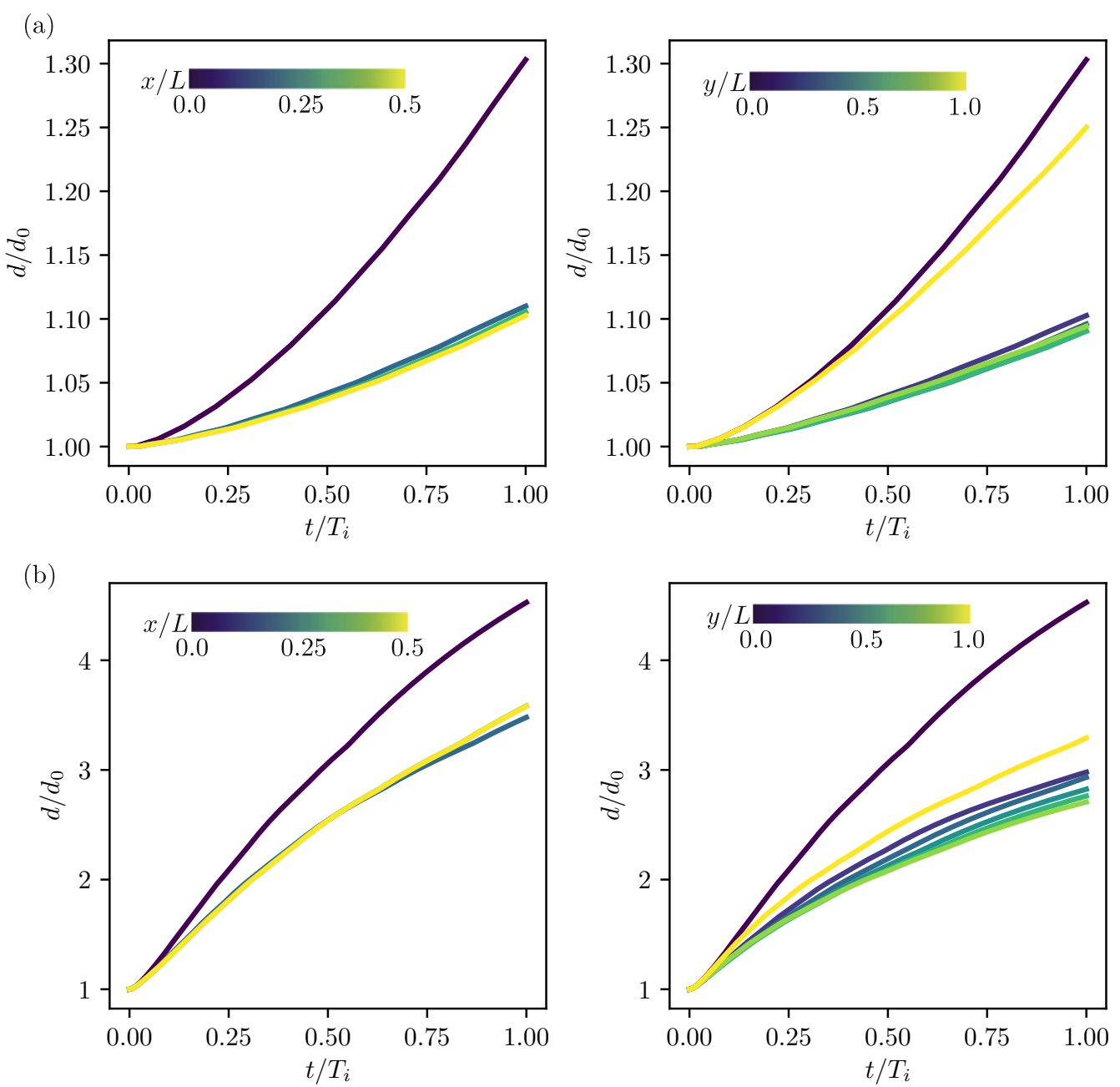

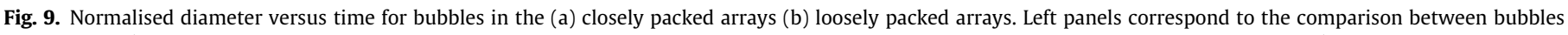

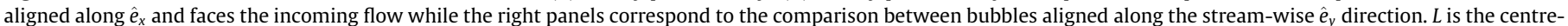

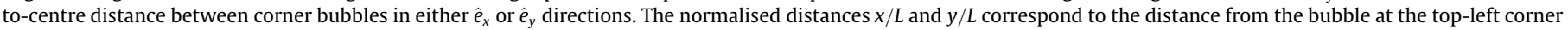
in Fig. 8. 
(a)



(b)

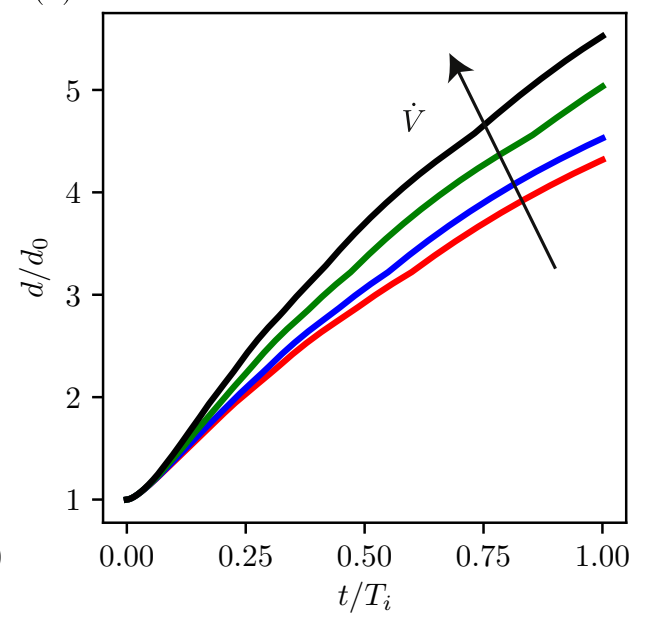

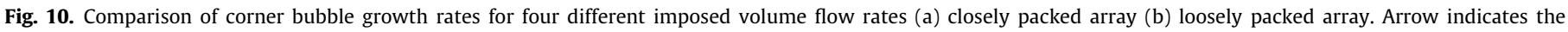
direction of increasing volume flow rate.

arrays. In the case of closely packed arrays, we observe that similar to the experimental measurements, the corner bubbles grow much more rapidly than that of the other bubbles given that they have a relatively easier access to over-saturation. In the case of loosely packed bubbles, the growth of corner bubbles in comparison to other bubbles is much weaker given the fact that the incoming flow faces much lower resistance to pass through the array and thus consequently enhancing solvent exchange with all bubbles in the array.

We now quantify the growth of bubbles by comparing the diameter of the bubbles as seen along $\hat{e}_{z}$ relative to its initial diameter $\left(d_{0}\right)$. In Fig. 9, we plot the normalised diameter of individual drops along the span-wise $\left(\hat{e}_{x}\right)$ and stream-wise $\left(\hat{e}_{y}\right)$ directions for both closely packed and loosely packed arrays. In the case of closely packed arrays (Fig. 9(a)), the corner bubbles grow much more rapidly than the remaining bubbles. In the case of loosely packed arrays the bubbles grow more homogeneously in comparison to the closely packed arrays.

In Fig. 10, we show the influence of the volume flow rate on the growth rates of the corner bubble for both the closely packed and loosely packed arrays, respectively. In both cases, we observe that the growth rate of the bubble is increased with increasing volume flow rates, which is consistent with the experimental results shown in Figs. 3 and 6. It also should be noted that, at the same solvent exchange flow rate condition, the simulated bubble growth rate for bubbles located on the corners of loosely packed arrays is much faster than that on the closely packed arrays. Taking the flow rate of $200 \mu \mathrm{L} / \mathrm{min}$ as an example, the overall bubble growth rate for the closely and loosely packed arrays were $0.142 \mu \mathrm{m} / \mathrm{s}$ and $0.277 \mu \mathrm{m} / \mathrm{s}$ respectively. Under the same flow rate condition, the growth rates for bubbles located at the corners can reach $1.3 \mu \mathrm{m} / \mathrm{s}$ and $4.5 \mu \mathrm{m} / \mathrm{s}$ respectively based on the numerical simulation in Fig. 10. The enhanced bubble growth rates can be primarily attributed by the edge/corner effect, which is due to the relatively higher oversaturation level at these edge/corner locations.

We note that results from DNS are not limited to the real dimensions in our experiments, but applicable to bubbles with different sizes and spatial arrangements. We expect the effects of flow rate and location of nucleation sites may also hold for surface nanobubbles produced by solvent exchange, although it remains challenging to verify the diffusive dynamics on that small scale in experiments.

\section{Conclusions}

On the basis of the previously reported approaches to generate surface nanobubbles and nanodroplets [33,26,35,34,24], this work has demonstrated that it is feasible to control the formation and growth of microbubbles on a cavity surface over a large area by a simple solvent exchange process and numerically simulate the essence of this process. We investigated the diffusive growth dynamics of microbubbles on cavity arrays during solvent exchange. The growth rates and number densities of the bubbles are closely related to the flow rate and spatial arrangement of the cavities. Our experimental results show that higher bubble growth rates were obtained when faster solvent exchange flow rates were applied. At the same time, more cavities were activated for surface microbubbles to grow. The edge effect on the cavity array substrates during bubble growth was considerately reduced as a result of higher gas oversaturation levels induced by higher flow rates. On the contrary, under the same solvent exchange flow rate, bubble growth rates decreased when the concentration of ethanol solution for solvent exchange was decreased from $100 \%$ to $70 \%$. No obvious edge effects were observed when the distance between cavities was increased from $13 \mu \mathrm{m}$ to $40 \mu \mathrm{m}$. The collective effects from growing bubbles in proximity promote larger bubbles at the edges and corners of the arrays, due to the influence of the flow rate. The main features of the bubble growth behaviour in experiments are in agreement with the results from numerical simulations of the diffusion equation, to which the growing bubbles are coupled with the immersed boundary method. The findings in this work can also be extended to the diffusive interaction of neighboring surface nanobubbles during solvent exchange.

\section{Acknowledgments}

S.P. is from an ARENA Research Fellowship and X.H.Z. acknowledges the support from Australian Research Council (LP140100594). We thank Dr. Shuying Wu for technical assistance in SEM imaging and Martin Klein Schaarsberg for assistance in data analysis. We also acknowledge the use of facilities and the associated technical support at the RMIT MicroNano Reserach Facility (MNRF) and the Microscopy and Microanalysis Facility (RMMF). This work was partly supported by the Netherlands Center for Multiscale Catalytic Energy Conversion (MCEC), an NWO 
Gravitation programme funded by the Ministry of Education, Culture and Science of the government of the Netherlands. The simulations were carried out on the national e-infrastructure of SURFsara, a subsidiary of SURF cooperation, the collaborative ICT organization for Dutch education and research. We also acknowledge PRACE for awarding us access to Marconi super computer, based in Italy at CINECA under PRACE project number 2016143351.

\section{References}

[1] D. Lohse, X. Zhang, Surface nanobubbles and nanodroplets, Rev. Mod. Phys. 87 (2015) 981-1035.

[2] V.S.J. Craig, Very small bubbles at surfaces-the nanobubble puzzle, Soft Matter 7 (1) (2011) 40-48.

[3] C.C. Chan, C.-D. Ohl, Total-internal-reflection-fluorescence microscopy for the study of nanobubble dynamics, Phys. Rev. Lett. 109 (2012) 174501.

[4] Q. Chen, H.S. Wiedenroth, S.R. German, H.S. White, Electrochemical nucleation of stable N2 nanobubbles at pt nanoelectrodes, J. Am. Chem. Soc 137 (37) (2015) 12064-12069.

[5] C. Huang, J. Jiang, M. Lu, L. Sun, E.I. Meletis, Y. Hao, Capturing electrochemically evolved nanobubbles by electroless deposition. A facile route to the synthesis of hollow nanoparticles, Nano Lett. 9 (12) (2009) 4297-4301.

[6] H. An, G. Liu, R. Atkin, V.S. Craig, Surface nanobubbles in nonaqueous media: looking for nanobubbles in dmso, formamide, propylene carbonate, ethylammonium nitrate, and propylammonium nitrate, ACS Nano 9 (7) (2015) 7596-7607.

[7] P. Attard, The stability of nanobubbles, Eur. Phys. J. Spec. Top. (2013) 1-22.

[8] W.A. Ducker, Contact angle and stability of interfacial nanobubbles, Langmuir 25 (16) (2009) 8907-8910.

[9] M. Guan, W. Guo, L. Gao, Y. Tang, J. Hu, Y. Dong, Investigation on the temperature difference method for producing nanobubbles and their physical properties, ChemPhysChem 13 (8) (2012) 2115-2118.

[10] S.R. German, M.A. Edwards, H. Ren, H.S. White, Critical nuclei size, rate, and activation energy of H2 gas nucleation, J. Am. Chem. Soc. 140 (2018) 40474053.

[11] J.W. Tyrrell, P. Attard, Images of nanobubbles on hydrophobic surfaces and their interactions, Phys. Rev. Lett. 87 (17) (2001) 176104.

[12] W. Bouwhuis, R.C. van der Veen, T. Tran, D.L. Keij, K.G. Winkels, I.R. Peters, D. van der Meer, C. Sun, J.H. Snoeijer, D. Lohse, Maximal air bubble entrainment at liquid-drop impact, Phys. Rev. Lett. 109 (26) (2012) 264501.

[13] O. Chen, L. Luo, H. Faraji, S.W. Feldberg, H.S. White, Electrochemica measurements of single $\mathrm{H} 2$ nanobubble nucleation and stability at pt nanoelectrodes, J. Phys. Chem. Lett. 5 (20) (2014) 3539-3544.

[14] A.C. Simonsen, P.L. Hansen, B. Klösgen, Nanobubbles give evidence of incomplete wetting at a hydrophobic interface, J. Colloid Interface Sci. 273 (1) (2004) 291-299.

[15] J.W.G. Tyrrell, P. Attard, Atomic force microscope images of nanobubbles on a hydrophobic surface and corresponding force- separation data, Langmuir 18 (1) (2002) 160-167.

[16] G. Shen, X.H. Zhang, Y. Ming, L. Zhang, Y. Zhang, J. Hu, Photocatalytic induction of nanobubbles on TiO2 surfaces, J. Phys. Chem. C 112 (11) (2008) 4029-4032.

[17] M. Hampton, A. Nguyen, Nanobubbles and the nanobubble bridging capillary force, Adv. Colloid Interface Sci. 154 (1) (2010) 30-55.

[18] F.Y. Ushikubo, T. Furukawa, R. Nakagawa, M. Enari, Y. Makino, Y. Kawagoe, T. Shiina, S. Oshita, Evidence of the existence and the stability of nano-bubbles in water, Colloids Surf. A 361 (1-3) (2010) 31-37.

[19] W. Tsai, P. Hsu, Y. Hwu, C. Chen, L. Chang, J. Je, H. Lin, A. Groso, G. Margaritondo, Building on bubbles in metal electrodeposition, Nature 417 (6885) (2002) 139.

[20] L. Luo, H.S. White, Electrogeneration of single nanobubbles at sub-50-nmradius platinum nanodisk electrodes, Langmuir 29 (35) (2013) 11169-11175.
[21] X. Zhang, D.Y.C. Chan, D. Wang, N. Maeda, Stability of interfacial nanobubbles, Langmuir 29 (4) (2013) 1017-1023.

[22] C. Xu, S. Peng, G.G. Qiao, V. Gutowski, D. Lohse, X. Zhang, Nanobubble formation on a warmer substrate, Soft Matter 10 (39) (2014) 7857-7864.

[23] A. Habich, W. Ducker, D.E. Dunstan, X. Zhang, Do stable nanobubbles exist in mixtures of organic solvents and water?, J Phys. Chem. B 114 (20) (2010) 6962-6967.

[24] X.H. Zhang, A. Khan, W.A. Ducker, A nanoscale gas state, Phys. Rev. Lett. 98 (13) (2007) 136101.

[25] M. Liu, W. Zhao, S. Wang, W. Guo, Y. Tang, Y. Dong, Study on nanobubble generation: saline solution/water exchange method, ChemPhysChem 14 (11) (2013) 2589-2593.

[26] M.A. Hampton, B.C. Donose, A.V. Nguyen, Effect of alcohol-water exchange and surface scanning on nanobubbles and the attraction between hydrophobic surfaces, J. Colloid Interface Sci. 325 (1) (2008) 267-274.

[27] S.-T. Lou, Z.-Q. Ouyang, Y. Zhang, X.-J. Li, J. Hu, M.-Q. Li, F.-J. Yang, Nanobubbles on solid surface imaged by atomic force microscopy, J. Vac. Sci. Technol., B 18 (5) (2000) 2573-2575.

[28] S. Yang, E. Kooij, B. Poelsema, D. Lohse, H. Zandvliet, Correlation between geometry and nanobubble distribution on hopg surface, EPL 81 (6) (2008) 64006.

[29] G. Pan, B. Yang, Effect of surface hydrophobicity on the formation and stability of oxygen nanobubbles, ChemPhysChem 13 (8) (2012) 2205-2212.

[30] W. Guo, H. Shan, M. Guan, L. Gao, M. Liu, Y. Dong, Investigation on nanobubbles on graphite substrate produced by the water- $\mathrm{NaCl}$ solution replacement, Surf. Sci. 606 (17-18) (2012) 1462-1466.

[31] X. Wang, B. Zhao, W. Ma, Y. Wang, X. Gao, R. Tai, X. Zhou, L. Zhang, Interfacial nanobubbles on atomically flat substrates with different hydrophobicities, ChemPhysChem 16 (5) (2015) 1003-1007.

[32] Z. Lu, S. Peng, X. Zhang, Influence of solution composition on the formation of surface nanodroplets by solvent exchange, Langmuir 32 (7) (2016) 1700-1706.

[33] S. Peng, T.L. Mega, X. Zhang, Collective effects in microbubble growth by solvent exchange, Langmuir 32 (43) (2016) 11265-11272.

[34] X. Zhang, Z. Lu, H. Tan, L. Bao, Y. He, C. Sun, D. Lohse, Formation of surface nanodroplets under controlled flow conditions, Proc. Natl. Acad. Sci. USA 112 (2015) 9253-9257.

[35] B. Dyett, A. Kiyama, M. Rump, Y. Tagawa, D. Lohse, X. Zhang, Growth dynamics of surface nanodroplets during solvent exchange at varying flow rates, Soft Matter 14 (8) (2018) 5197-5204.

[36] L. Bao, V. Spandan, Y. Yang, B. Dyett, R. Verzicco, D. Lohse, X. Zhang, Flowinduced dissolution of femtoliter surface droplet arrays, Lab on a Chip 18 (7) (2018) 1066-1074.

[37] X. Zhu, R. Verzicco, X. Zhang, D. Lohse, Diffusive interaction of multiple surface nanobubbles: shrinkage, growth, and coarsening, Soft Matter 14 (2018) 20062014.

[38] R. Mittal, G. Iaccarino, Immersed boundary methods, Annu. Rev. Fluid Mech. 37 (2005) 239-261.

[39] V. Spandan, V. Meschini, R. Ostilla-Mónico, D. Lohse, G. Querzoli, M.D. de Tullio, R. Verzicco, A parallel interaction potential approach coupled with the immersed boundary method for fully resolved simulations of deformable interfaces and membranes, J. Comp. Phys. 348 (2017) 567-590.

[40] M.D. de Tullio, G. Pascazio, A moving-least-squares immersed boundary method for simulating the fluid-structure interaction of elastic bodies with arbitrary thickness, J. Comp. Phys. 325 (2016) 201-225.

[41] R. Ostilla-Monico, Y. Yang, E.P. van der Poel, D. Lohse, R. Verzicco, A multipleresolution strategy for direct numerical simulation of scalar turbulence, J. Comp. Phys. 301 (2015) 308-321.

[42] C. Xu, H. Yu, S. Peng, Z. Lu, L. Lei, D. Lohse, X. Zhang, Collective interactions in the nucleation and growth of surface droplets, Soft Matter 13 (2017) 937-944.

[43] L. Bao, Z. Werbiuk, D. Lohse, X. Zhang, Controlling the growth modes of femtoliter sessile droplets nucleating on chemically patterned surfaces, J. Phys. Chem. Lett. 7 (6) (2016) 1055-1059. 